Supplement of Earth Syst. Dynam., 10, 617-630, 2019

https://doi.org/10.5194/esd-10-617-2019-supplement

(C) Author(s) 2019. This work is distributed under

the Creative Commons Attribution 4.0 License.

Earth System
Dynamics

(c) (1)

Supplement of

\title{
Modeling forest plantations for carbon uptake with the LPJmL dynamic global vegetation model
}

Maarten C. Braakhekke et al.

Correspondence to: Maarten C. Braakhekke (maarten.braakhekke@gmail.com)

The copyright of individual parts of the supplement might differ from the CC BY 4.0 License. 


\section{Supplemental information}

\section{S1. Target growth curve derivation}

The growth curves for the three FPFTs were derived from observations and LPJmL predictions for the corresponding natural PFTs using Bayesian non-linear regression. We assume that observed stemwood $C$ is given by:

$$
C_{\mathrm{SWC}}^{\mathrm{obs}}(t)=C_{\mathrm{SWC}}^{\mathrm{pred}}(t)+\varepsilon_{t}=C_{\mathrm{SWC}, \max }\left(1-e^{-k t}\right)^{p}+\varepsilon_{t},
$$

where $\varepsilon_{t}$ is the model-data residual, assumed to be stochastic. We used normal priors for the parameters of the growth curve. For $C_{\mathrm{SWC} \text {,max }}$ strong priors were used, based on maximum stemwood C for the corresponding natural PFTs predicted by LPJmL (see section 2.3.1 in the main text; $N(5.43,1.88), N(14.0,2.40)$, and $N(6.16,1.24)$ for the temperate, tropical and boreal FPFT, respectively). For $k$ and $p$ weaker priors were used $(N(0.05,1)$ and $N(2,20)$, respectively, for all three FPFTs), allowing them to be more strongly influenced by the observations. Model-data residuals can be expected to scale with stemwood $C$, since both predictions and observations cannot be lower than zero. This is exacerbated by the fact that we incorporate a strong prior for $C_{\mathrm{SWC} \text {,max }}$, which may to some extent be inconsistent with the data, leading to a stronger misfit for higher plantation ages. Ignoring this heteroscedasticity may result in biased parameter estimates. Therefore we assumed that the variance of $\varepsilon_{t}$ scales linearly with predicted stemwood C:

$$
\varepsilon_{t} \sim N\left(0, \sigma_{s} C_{\mathrm{SWC}}^{\mathrm{pred}}(t)\right)
$$

where $\sigma_{s}$ is a scaling factor, simultaneously determined in the fitting, using a weak prior $(N(1,10))$.

The fitting was carried out with Markov Chain Monte Carlo sampling, implemented in the Stan programming language (Carpenter et al., 2017), linked to Matlab (Stan Development Team, 2017). Specifically, we used the No-U-Turn-Sampler (NUTS; Hoffman and Gelman, 2014) with four chains of 10,000 samples each, and a warmup period of 10,000 iterations per chain. Figure S1 depicts the marginal posterior distributions of the four estimated parameters for the three FPFTs, together with the prior distributions. The parameter set with highest posterior density, together with variance over the complete sample, was used for the target growth curve in the LPJmL calibration (see table S2). 

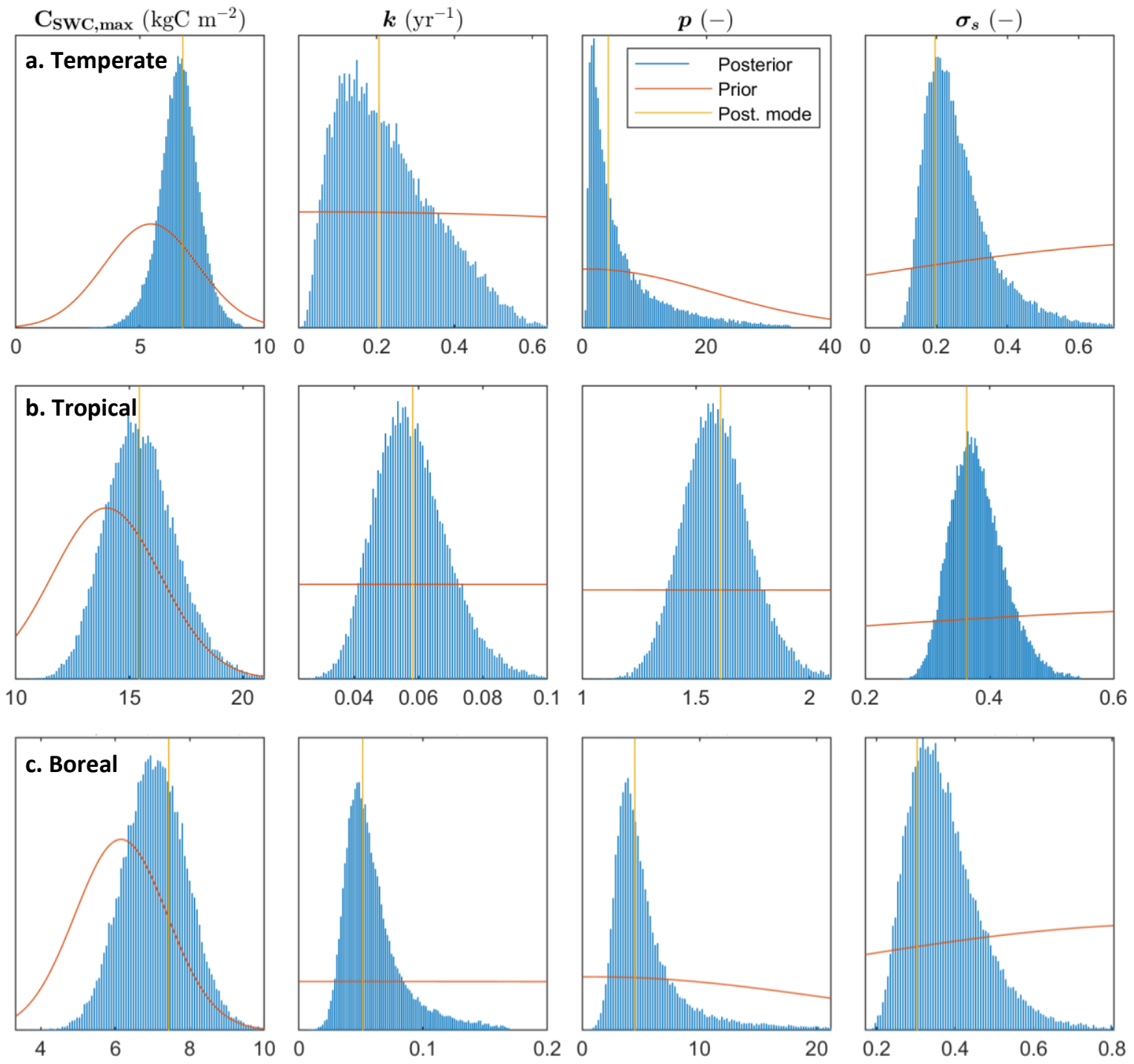

Figure S1. Results of the Monte Carlo sampling to determine the parameters of the target growth curves for temperate (a), tropical (b), and boreal (c) forest plantations. Note that the multivariate posterior mode, determined as the sample with highest posterior density (yellow lines in the graphs), deviates from the marginal modes (peak of the histograms), due to non-linear parameter correlations. 


\section{S2. LPJmL calibration}

The optimization of the FPFT parameters in this study comprised minimization of a Bayesian costfunction, essentially negative log posterior density. The cost-function is defined as he sum of a loglikelihood term and log prior density term. Prior distributions on the parameters were defined by gaussian probability density functions, defined on logit scale, for $\alpha_{a}$, and log-scale for all other parameters. Similarly, the observations were log- or logit-transformed as well. For a given parameter set $\theta$ the overall cost $C(\theta)$ is given by:

$$
C(\theta)=\sum_{i=1}^{I} \frac{\left(f_{i}\left(\theta_{i}\right)-\mu_{\theta_{i}}\right)^{2}}{\sigma_{\theta_{i}}^{2}}+\sum_{j=1}^{J} \frac{\left(f_{j}\left(\varphi_{j}\right)-\mu_{\varphi_{j}}\right)^{2}}{\sigma_{\varphi_{j}}^{2}},
$$

The first term on the right-hand size of (3) represents the prior in which: $I$ is the number of optimized parameters (15), $\theta_{i}$ and $f_{i}$ are the value and the transformation function (logit or log) of parameter $i$, and $\mu_{\theta_{i}}$ and $\sigma_{\theta_{i}}^{2}$ are the mean and variance of the prior for parameter $i$ on transformed scale. Additionally, bounds were enforced on the parameters. The lower bound was at 0 and the upper bound at the $99 \%$ quantile of the prior distributions, except for $\alpha_{a}$, for which it was set at 1 . Table S1 shows $\mu_{\theta_{i}}, \sigma_{\theta_{i}}^{2}$, and the upper bound of all parameters for the three FPFTs. The second term on the righthand size of (3) represents the likelihood, in which: $J$ is the number of observations (5), $\varphi_{j}$ and $f_{j}$ are the LPJmL output (based on parameter set $\theta$ ) and transformation function (logit or log) of observation variable $j$, and $\mu_{\varphi_{j}}$ and $\sigma_{\varphi_{j}}^{2}$ are the mean and variance of observation variable $j$ on transformed scale. Table S2 shows the $\mu_{\varphi_{i}}, \sigma_{\varphi_{i}}^{2}$ for all observations for the three FPFTs.

Table S1. Mean $(\mu)$, variance $\left(\sigma^{2}\right)$, and upper bound (u.b.) of the prior distributions on the parameters used in the LPJmL calibration. Note that $\mu$ and $\sigma^{2}$ are defined on transformed (log or logit) scale, while the upper bound is defined on untransformed scale.

\begin{tabular}{|c|c|c|c|c|c|c|c|c|c|c|}
\hline \multirow{2}{*}{$\begin{array}{l}\text { Parameter } \\
\text { (i) }\end{array}$} & \multirow{2}{*}{$\begin{array}{c}\text { Transfor } \\
\text { mation } \\
\left(f_{i}\right)\end{array}$} & \multicolumn{3}{|c|}{ Temperate } & \multicolumn{3}{|c|}{ Tropical } & \multicolumn{3}{|c|}{ Boreal } \\
\hline & & $\boldsymbol{\mu}_{\boldsymbol{\theta}_{i}}$ & $\sigma_{\theta_{i}}^{2}$ & u.b. & $\boldsymbol{\mu}_{\boldsymbol{\theta}_{i}}$ & $\sigma_{\theta_{i}}^{2}$ & u.b. & $\mu_{\theta_{i}}$ & $\sigma_{\theta_{i}}^{2}$ & u.b. \\
\hline$\alpha_{a}$ & logit & 0.00 & 0.700 & 1.00 & 0.00 & 0.700 & 1.00 & 0.00 & 0.700 & 1.00 \\
\hline$g_{\min }$ & $\log$ & -0.693 & 0.300 & 2.41 & -0.693 & 0.300 & 2.41 & -1.20 & 0.300 & 1.45 \\
\hline$E_{\text {max }}$ & $\log$ & 1.61 & 0.300 & 24.1 & 1.95 & 0.300 & 33.8 & 1.61 & 0.300 & 24.1 \\
\hline$r$ & $\log$ & 0.182 & 0.300 & 5.79 & -1.61 & 0.300 & 0.965 & 0.182 & 0.300 & 5.79 \\
\hline$k_{\text {allom1 }}$ & $\log$ & 4.61 & 0.300 & 481 & 4.61 & 0.300 & 4.61 & 4.70 & 0.300 & 530 \\
\hline $\boldsymbol{k}_{\text {allom2 }}$ & $\log$ & 3.69 & 0.300 & 193 & 3.69 & 0.300 & 193 & 3.69 & 0.300 & 193 \\
\hline $\boldsymbol{k}_{\text {allom3 }}$ & $\log$ & -0.400 & 0.300 & 3.23 & -0.40 & 0.300 & 3.23 & -0.40 & 0.300 & 3.23 \\
\hline $\mathbf{l} \mathbf{r}_{\max }$ & $\log$ & 0.00 & 0.300 & 4.83 & 0.00 & 0.300 & 4.83 & 0.00 & 0.300 & 4.83 \\
\hline $\mathrm{C}_{\text {sapwood;sapl }}$ & $\log$ & 0.182 & 0.300 & 5.79 & 0.182 & 0.300 & 5.79 & 0.182 & 0.300 & 5.79 \\
\hline $\mathbf{L A I}_{\text {sapl }}$ & $\log$ & 0.405 & 0.300 & 7.24 & 0.405 & 0.300 & 7.24 & 0.405 & 0.300 & 7.24 \\
\hline$\alpha_{\text {leaf }}$ & $\log$ & -0.693 & 0.300 & 2.41 & 0.693 & 0.300 & 9.65 & 1.39 & 0.300 & 19.3 \\
\hline$\tau_{\text {sapwood }}$ & $\log$ & 3.00 & 0.300 & 96.5 & 3.00 & 0.300 & 96.5 & 3.00 & 0.300 & 96.5 \\
\hline$\tau_{\text {leaf,root }}$ & $\log$ & 0.00 & 0.300 & 4.83 & 0.693 & 0.300 & 9.65 & 1.39 & 0.300 & 19.3 \\
\hline$P_{\text {init }}$ & $\log$ & -1.90 & 0.300 & 0.724 & -1.90 & 0.300 & 0.724 & -1.90 & 0.300 & 0.724 \\
\hline$k_{\text {mort1 }}$ & $\log$ & -3.51 & 0.300 & 0.145 & -3.51 & 0.300 & 0.145 & -3.51 & 0.300 & 0.145 \\
\hline
\end{tabular}


Table S2. Mean $(\mu)$, variance $\left(\sigma^{2}\right)$, of the likelihood functions for the observations used in the LPJmL calibration. Note that $\mu$ and $\sigma^{2}$ are defined on transformed (log or logit) scale.

\begin{tabular}{|c|c|c|c|c|c|c|c|}
\hline \multirow{2}{*}{$\begin{array}{l}\text { Obser- } \\
\text { vation } \\
\text { (j) }\end{array}$} & \multirow{2}{*}{$\begin{array}{l}\text { Transfor- } \\
\text { mation } \\
\left(f_{j}\right)\end{array}$} & \multicolumn{2}{|c|}{ Temperate } & \multicolumn{2}{|c|}{ Tropical } & \multicolumn{2}{|c|}{ Boreal } \\
\hline & & $\boldsymbol{\mu}_{\varphi_{j}}$ & $\sigma_{\varphi_{j}}^{2}$ & $\mu_{\varphi_{j}}$ & $\sigma_{\varphi_{j}}^{2}$ & $\mu_{\varphi_{j}}$ & $\sigma_{\varphi_{j}}^{2}$ \\
\hline $\mathrm{C}_{\mathrm{SWC}, \mathrm{max}}$ & $\log$ & 8.82 & 0.0163 & 9.66 & 0.00989 & 8.92 & 0.0150 \\
\hline $\boldsymbol{k}$ & $\log$ & -1.63 & 0.431 & -2.87 & 0.0389 & -3.00 & 0.300 \\
\hline$p$ & $\log$ & 1.36 & 0.865 & 0.465 & 0.00889 & 1.45 & 0.164 \\
\hline CUE & logit & -0.488 & 0.200 & -0.169 & 0.0262 & -0.159 & 0.123 \\
\hline$\tau_{\mathrm{vegC}}$ & $\log$ & 2.82 & 0.0500 & 3.09 & 0.0500 & 3.10 & 0.05 \\
\hline
\end{tabular}

The parameter set $\theta$ at which the cost is minimal was obtained using the genoud (GENetic Optimization Using Derivatives) algorithm. Genoud is a hybrid optimization scheme that combines a genetic algorithm with gradient-search approach (Sekhon and Mebane, 1998). Genetic algorithms are schemes that search for solutions to optimization problems with complex, non-smooth functions. These problems are often difficult to solve using gradient-search algorithms (e.g. Newton-Raphson), which rely on derivatives in order to determine successive steps. Genetic algorithms use an approach inspired by the process of natural selection, working with a population of candidate solutions (parameter sets), which are evolved in successive generations. This is done by means of "genetic operators", which combine and mutate individuals in the population. While genetic algorithms are highly suitable for irregular functions, they are often slower than gradient-search algorithms in the neighborhood of the global solution, where the surface is usually smooth and concave. Therefore, genoud employs a gradient-search algorithm on the best candidate solution in the population after each generation. The parameter value resulting from this gradient-search step is used in next generation. The algorithm is implemented in R language and available as the rgenoud package (Mebane Jr. and Sekhon, 2011). In our calibrations we used a population size of 1000 individuals and a maximum number of 20 generations. For the gradient-search algorithm the BGFS scheme was used. 

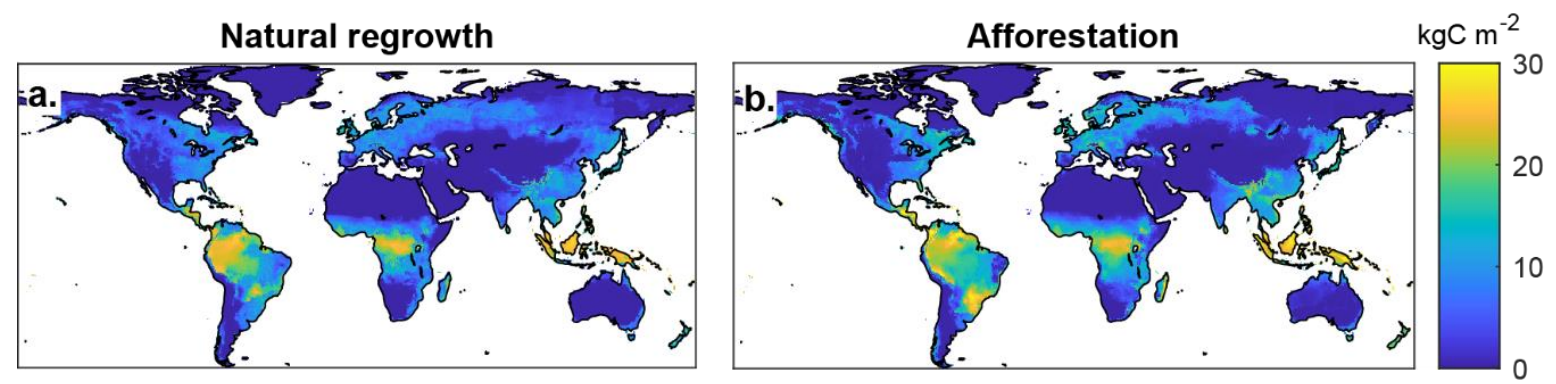

Figure S2. Total ecosystem C after 300 years for a global simulation with only natural vegetation and only forest plantations.

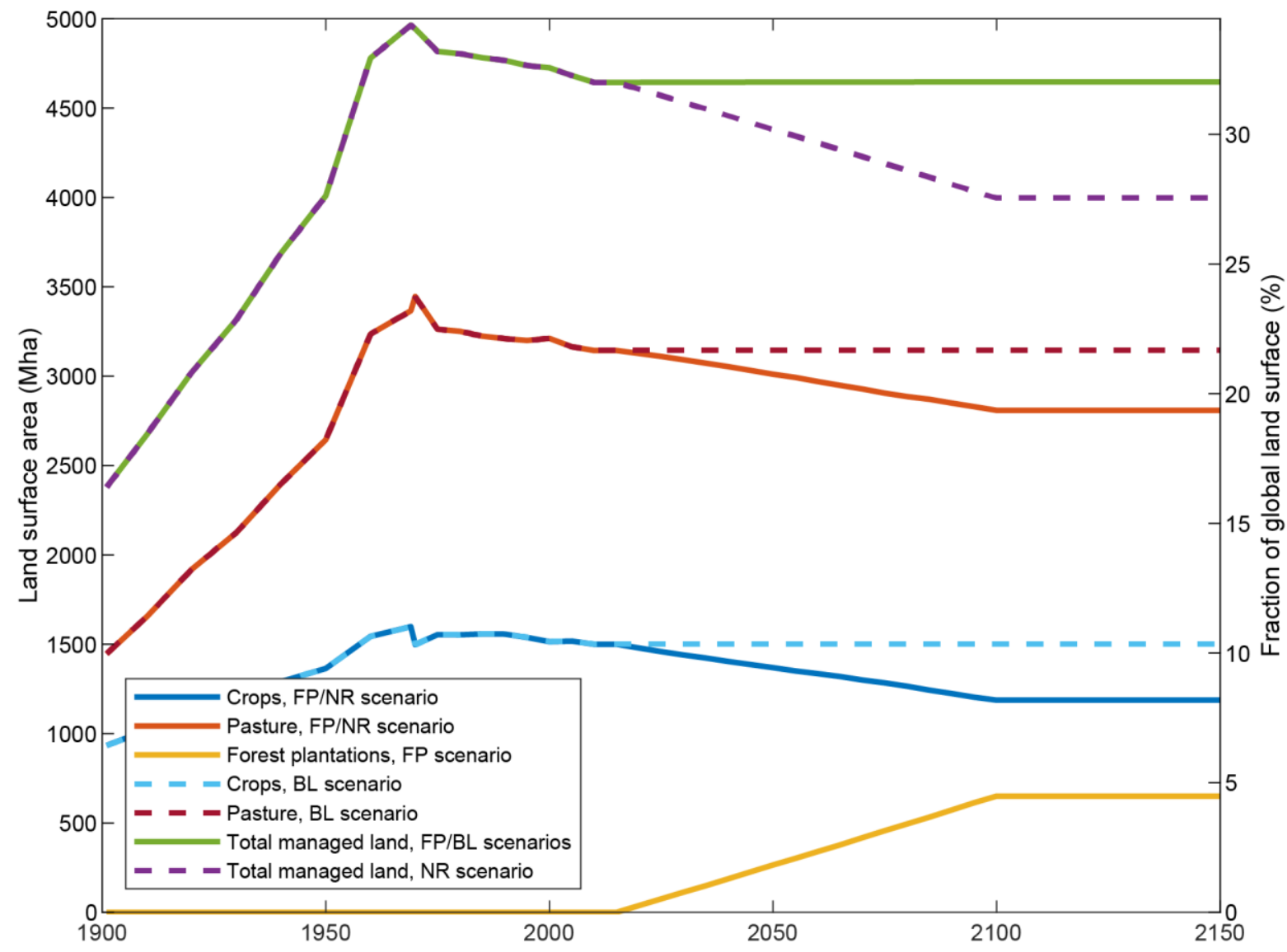

Figure S3. Development of land-use fractions for the transient afforestation scenario (FP) and accompanying scenarios for natural regrowth (NR) and the baseline (BL) with constant land-use after 2015. "Total managed land" refers to the sum of croplands, pasture, and forest plantations. 


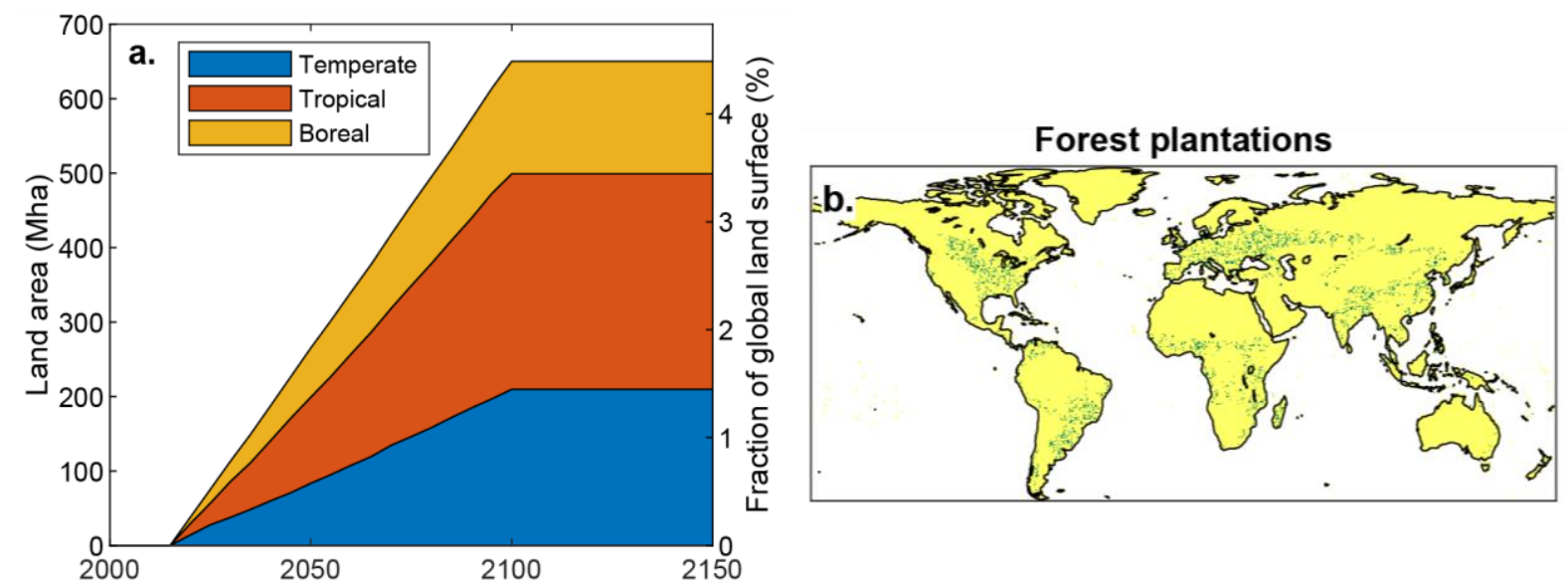

Figure S4. Forest plantation land use fraction for the transient afforestation scenario. (a) global total afforestation area versus time; (b) afforested grid cells from 2100. 